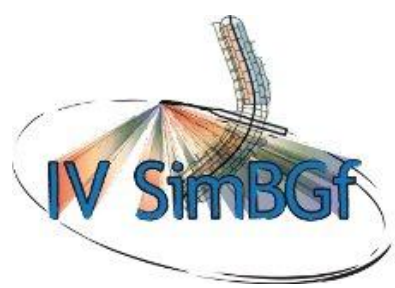

\title{
ANÁLISE DO POTENCIAL ELÉTRICO NATURAL GERADO EM ATERRO DE RESÍDUOS SÓLIDOS DOMICILIARES
}

César Augusto Moreira \& Aline Muriel da Cunha Menezes*

Universidade Federal do Pampa - UNIPAMPA. Avenida Pedro Anunciação, s/n, Vila Batista, CEP 96570-000, Caçapava do Sul - RS

Copyright 2010, SBGf - Sociedade Brasileira de Geofísica

Este texto foi preparado para a apresentação no IV Simpósio Brasileiro de Geofísica, Brasília, 14 a 17 de novembro de 2010. Seu conteúdo foi revisado pelo Comitê Brasília, 14 a 17 de novembro de 2010. Seu conteúdo foi revisado pelo Comitê
Técnico do IV SimBGf, mas não necessariamente representa a opinião da SBGf ou de seus associados. É proibida a reprodução total ou parcial deste material para propósitos comerciais sem prévia autorização da SBGf.

\begin{abstract}
This paper evaluates the results of natural electric potential together with $\mathrm{pH}$ and Eh measures in soil samples in waste landfill the ditch type. The landfill just receives domestic solid residues, willing in ditches with sequential opening and closing. The substratum of the area is constituted by loamy soil with $10 \mathrm{~m}$ of thickness, above for diabase body with $15 \mathrm{~m}$ of thickness. The results of natural electric potential indicate a relationship with the time of decomposition of the residues, in other words, gradational increase of the concomitant electric potential to the increase of the period of residence of the residues.
\end{abstract}

\section{Introdução}

Detectar alterações nas propriedades físicas provocadas pela percolação e residência de contaminantes em solos e rochas consiste no objeto de estudos da geofísica aplicada em estudos ambientais. O contraste entre áreas avaliadas e áreas próximas com características naturais ao parâmetro físico medido permite a delimitação 2D e 3D das áreas potencialmente contaminadas.

Torna-se crescente a necessidade de adaptação de aplicação de técnicas de investigação diretas e indiretas para uso em estudos ambientais devido a grande quantidade de compostos sintéticos ou derivados do petróleo para uso final ou como insumos para outras indústrias, riscos de derrame ou vazamento durante o transporte e manipulação destes compostos, além vazamentos ou descartes indevidos de efluentes e resíduos sólidos ou líquidos produzidos durante processos indústriais, atividades urbanas e domésticas.

Necessários e de caráter primordial são os estudos voltados a análise e compreensão do comportamento destes compostos no ambiente geológico, sob a ótica das alterações físicas e químicas resultantes da presença e interação com o meio, sazonalidades climáticas, disponibilidade de oxigênio, $\mathrm{pH}$, Eh, dentre outros. Tais alterações resultam em muitos casos, na redução da toxicidade dos compostos. Contudo, em alguns casos, pode ocorrer a liberação de metais eventualmente contidos nos constituintes minerais ou nos próprios compostos, além do eventual aumento da toxicidade de compostos gerados da degradação de contaminantes iniciais.

Compreender as modificações impressas nas propriedades físicas pela presença de contaminantes no ambiente geológico são essenciais à aplicação e interpretação da geofísica em estudos ambientais.

Os trabalhos de Naudet et al. (2003) e Naudet et al. (2004), utilizam medidas de potencial espontâneo concomitante a medidas de Eh, no estudo da evolução de uma pluma de contaminação proveniente de aterro sanitário.

Os resultados indicam que o potencial elétrico decresce progressivamente na zona aeróbica, aumenta drasticamente na frente de oxidação/redução e finalmente alcança o padrão da área de estudos ao atingir a zona de oxidação. Em perfil, há uma grande correlação entre potencial espontâneo negativo e medidas de potencial de oxidação/redução, com geração de potencial elétrico por processos de oxidação/redução dos compostos contaminantes.

Por meio de métodos geofísicos Elétricos e medidas de pH e Eh em amostras de solo, Moreira et al. (2009) objetivam avaliar o comportamento geoquímico de resíduos sólidos enterrados, durante o processo de degradação de matéria orgânica. Os estudos foram realizados em valas fechadas entre 12/2001 a 12/2007. Os resultados mostraram a presença de valores de resistividade abaixo de $30 \Omega . \mathrm{m}$ nas linhas realizadas sobre as valas, sugerindo a presença de chorume. A correlação temporal entre $\mathrm{pH}$ e Eh pode indicar as condições geoquímicas predominantes as quais estão associadas ao padrão de resistividade elétrica. Assim, baixos valores de resistividade associados a baixos valores de $\mathrm{pH}$ e elevados valores de Eh indicam a possível existência de uma pluma de contaminação.

Este trabalho avalia os resultados da aplicação do método de Potencial Espontâneo conjunto com medidas de $\mathrm{pH}$ e Eh, num aterro de resíduos orgânicos domiciliares, dispostos em valas com diversas datas de fechamento. A relação entre o tempo de decomposição dos resíduos orgânicos e as variações de voltagem medidas pelo método geofísico permitem estimar o tempo de estabilização geoquímica da área.

\section{Comportamento geoquímico do chorume no solo}

A decomposição de resíduos orgânicos em aterros por longos períodos envolve processos físico-químicos e 
químicos, notavelmente hidrólise, hidratação, carbonatação, oxidação e redução, além de degradação biológica - microbial, que resultam na dissolução e deterioração, geração de gás e produção de chorume.

O chorume pode conter substâncias tóxicas e perigosas sob a forma sólida, líquida ou gasosa e pode apresentar elevadas concentrações de cloreto, ferro e zinco. Os elementos com elevada mobilidade iônica geralmente apresentam altas concentrações em comparação aos elementos de baixa mobilidade iônica (Bagchi, 1987). T O $\mathrm{pH}$ tende a aumentar com o tempo, pois formas ácidas iniciais tendem a estados neutralizados, com decréscimo da Demanda Química por Oxigênio (DQO) e Demanda Biológica por Oxigênio (DBO).

\section{Caracterização da área de estudo}

O aterro de Cordeirópolis está localizado no km 4.5 da rodovia Cássio Freitas Levy, município de Cordeirópolis, Estado de São Paulo, Brasil (Figura 1). O município possui aproximadamente 17.000 habitantes e economia baseada na produção de revestimentos cerâmicos e cultivo de cana-de-açúcar. A produção diária de resíduos sólidos domiciliares e da ordem de $6 \mathrm{~m} 3$.

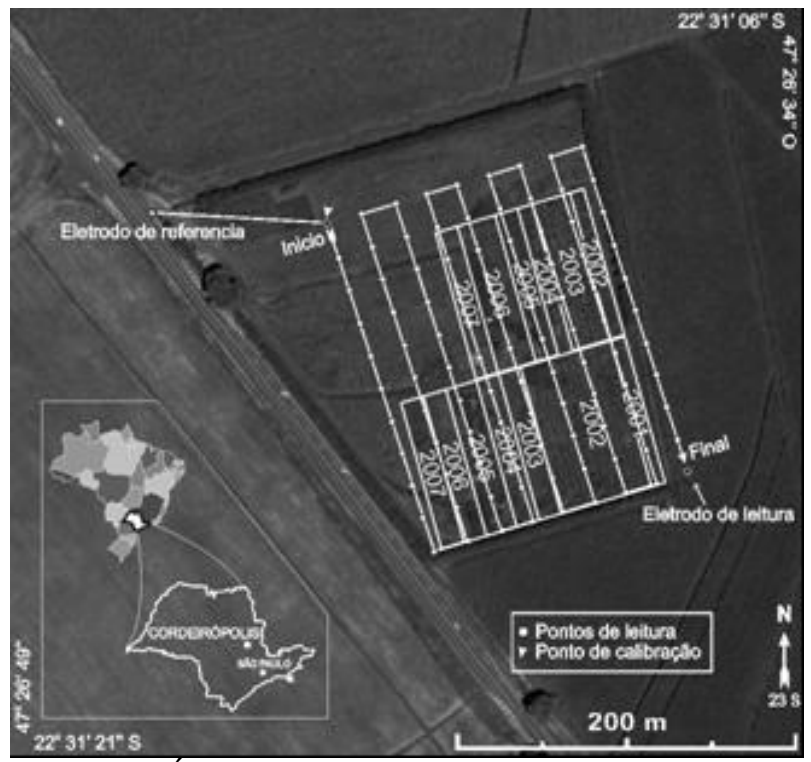

Figura 1 - Área de estudo.

A topografia da área é bastante plana, com declive médio de $0,5 \%$ sentido Sudeste e altitude entre $660 \mathrm{~m}$ e $659 \mathrm{~m}$. $\mathrm{O}$ aterro é cercado pelo cultivo de cana-de-açúcar.

O substrato é constituído por solo argiloso com 10m de espessura, sotoposto por um corpo de diabásio com $15 \mathrm{~m}$ de espessura. Abaixo deste ocorrem arenitos finos e siltitos pertencentes a Formação Tatuí.

Nível freático está localizado a uma profundidade media de 50m, com sentido de fluxo para sudeste, determinados por meio de sondagens elétricas verticais. Ensaios de condutividade hidráulica saturada de campo apontar valores médios de $10-4 \mathrm{~cm} / \mathrm{s}$ para o solo da área. Não existem poços instalados na área ou quaisquer outros sistemas de monitoramento ambiental.
Foram realizadas 241 leituras de potencial espontâneo por meio do arranjo de base fixa. O eletrodo de base foi fixado a montante do fluxo d'água subterrânea, precisamente no portão de acesso a área do aterro (Figura 3).

A aquisição foi realizada em duas etapas:

Primeira etapa: leituras de voltagem em malha quadrada de $20 \times 20 \mathrm{~m}$, para uma ampla caracterização da área.

Segunda etapa: leituras realizadas somente sobre as cavas com data de fechamento no mês de dezembro, entre 2001 e 2007, com espaçamento de $5 \mathrm{~m}$ entre leituras. Esta etapa objetivou determinar uma eventual relação a decomposição dos resíduos enterrados e variações de voltagem ao longo do tempo.

Este trabalho utilizou eletrodos não-polarizáveis de $\mathrm{Pb}$ $\mathrm{PbCl}$, caracterizados por elevada estabilidade ao longo do tempo (Petiau, 2000).

As medidas obtidas em malha regular foram georreferenciadas e interpoladas no programa Surfer 8.0, por meio do método de vizinhança (natural neighbor). As medidas realizadas sobre as valas datadas são apresentadas em gráfico.

No método de vizinhança, os grupos iniciais são determinados pelos mais altos coeficientes de associação mútua, ou seja, para a admissão de novos membros é suficiente determinar quais os representantes de maior coeficiente de associação com um dos elementos de determinado grupo (Landim, 2004).

Neste sentido, a escolha deste método de interpolação foi baseada na fidelidade aos valores interpolados e limitação da superfície aos valores extremos de entrada, visto não haver quaisquer tratamentos ou filtragens nos dados obtidos em campo.

\section{Método}

O método de potencial espontâneo é caracterizado pela realização de leituras de campo elétrico natural, sem necessidade de quaisquer circuitos de injeção de corrente elétrica ou geração de campo eletromagnético (Orellana, 1972).

Anomalias de potencial espontâneo resultam de variações de temperatura, gradiente de pressão, porosidade, migração de fluídos, variação de resistividade e da umidade do solo (Corwin, 1990). Quanto ao processo de geração, o potencial espontâneo pode ser classificado em potencial Eletroquímico, Eletrolítico e Mineral.

\section{Resultados e Discussão}

As medidas de potencial espontâneo em malha regular são apresentadas em mapa, sotopostas às idades de fechamento das valas de resíduos (Figura 2).

Os dados indicam valores mais negativos nas valas fechadas entre 2007 e 2006 (0mV a 10mV), e ampla ocorrência de valores entre $10 \mathrm{mV}$ e $30 \mathrm{mV}$ em valas com idades de fechamento entre 2005 e 2004. Medidas entre $30 \mathrm{mV}$ e $70 \mathrm{mV}$ ocorrem nas valas fechadas entre 2003 e 2001.

A posição no nível freático em torno de $50 \mathrm{~m}$ de profundidade, valas de resíduos escavadas a até $5 \mathrm{~m}$ de 
profundidade em solo argiloso, sugerem que as anomalias de potencial espontâneo sejam produzidas por atividade geoquímica. A relação entre valores de voltagem cada vez mais positivos com o aumento do tempo de degradação de resíduos orgânicos confirma está hipótese.

Medidas abaixo de $20 \mathrm{mV}$ estão praticamente contidas nos limites das valas fechadas entre 2007 e 2006. A tendência de valores positivamente crescentes nas valas mais antigas pode estar associada ao decréscimo de matéria orgânica disponível e a conseqüente estabilidade geoquímica.

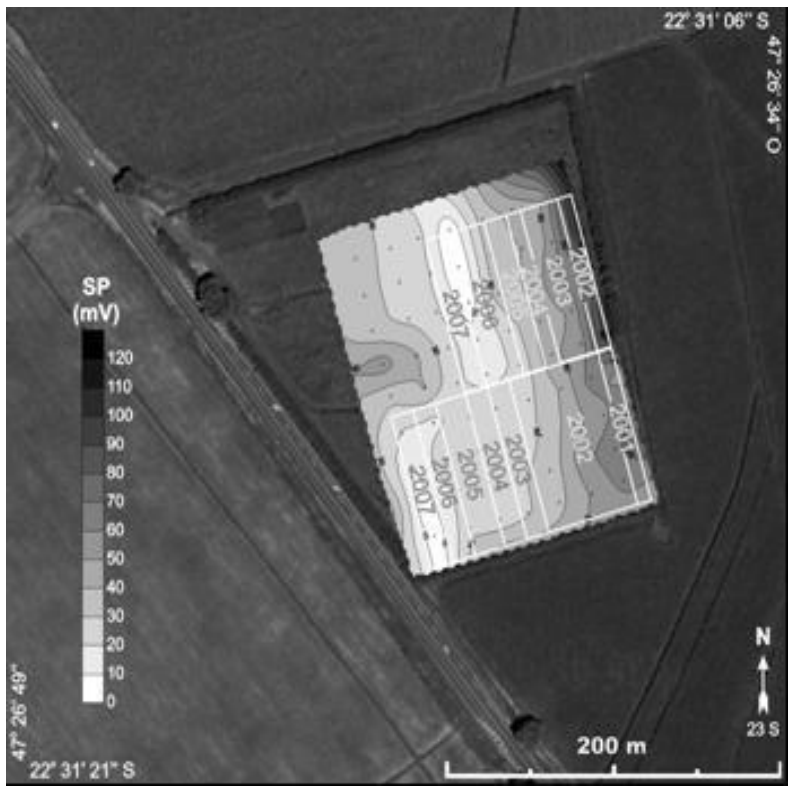

Figura 2 - Mapa de potencial espontâneo com a idade de fechamento das valas.

As medidas elevadas obtidas nas valas fechadas entre 2003 e 2001 podem indicar um avançado processo de degradação. Mesmo novas incursões de oxigênio gasoso a partir de fraturas de compactação no material de recobrimento das valas, resultam em pequena atividade geoquímica devido à redução drástica na oferta de matéria orgânica, consumida ao longo dos anos de residência dos resíduos.

Os dados de potencial espontâneo adquiridos durante a segunda etapa de levantamentos confirmam a tendência de aumento do potencial espontâneo das valas recentes para as valas antigas (Figura 3 e 4). Este aumento possivelmente está associado á estabilidade geoquímica crescente ao longo do período de degradação dos resíduos orgânicos.

Os valores obtidos sobre a linha de referência são relativamente pouco variáveis e superiores aos adquiridos sobre as valas de resíduos. Em contraponto, os valores obtidos sobre as valas apresentam tendência de aumento ou redução sentido ao final da linha, respectivamente referentes às valas de 2007 e 2003. Valores relativamente mais elevados na porção central das linhas são apresentados nas medidas obtidas sobre as valas de 2002 e 2001 .

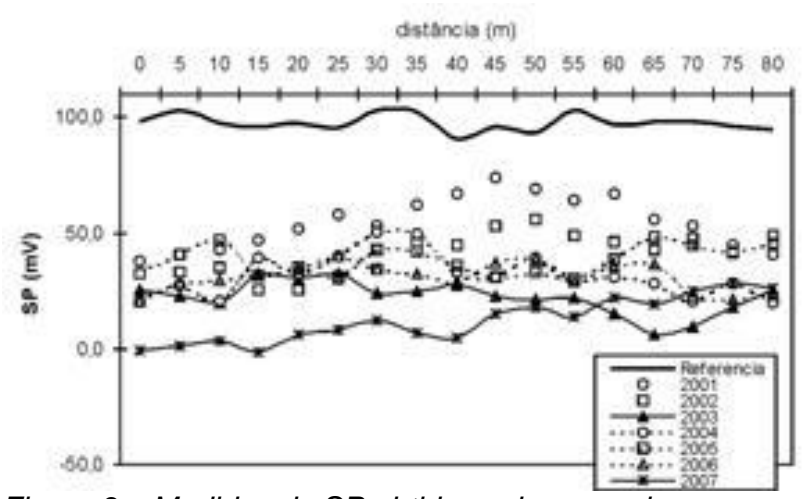

Figura 3 - Medidas de SP obtidas sobre as valas.

As medidas sobre a vala de 2007 apresentam relativamente os valores mais baixos, com medidas extremas entre $-1 \mathrm{mV}$ a $28 \mathrm{mV}$. As valas de 2006, 2005 e 2004 apresentam valores mais elevados, com extremos entre $20 \mathrm{mV}$ e $48 \mathrm{mV}$.

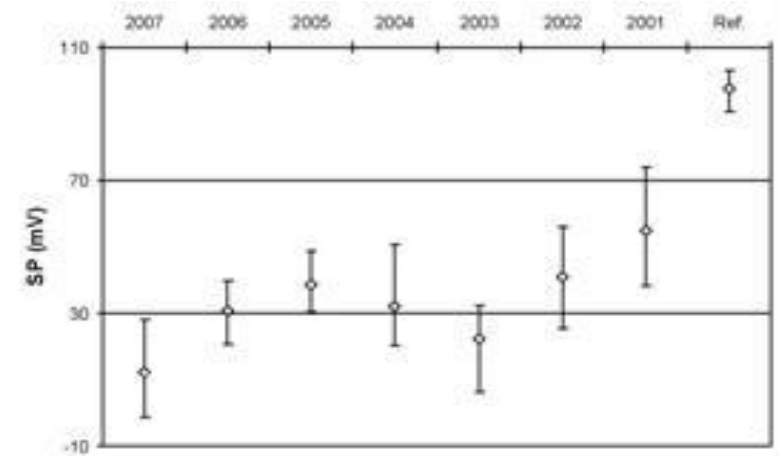

Figura 4 - Valores médios e extremos de SP da segunda etapa de levantamentos.

A análise estatística das medidas de potencial espontâneo, referente à média dos valores sobre as valas de resíduos, indica o período de oito anos para retorno aos valores naturais, a partir do primeiro ano de fechamento das valas (Figura 5).

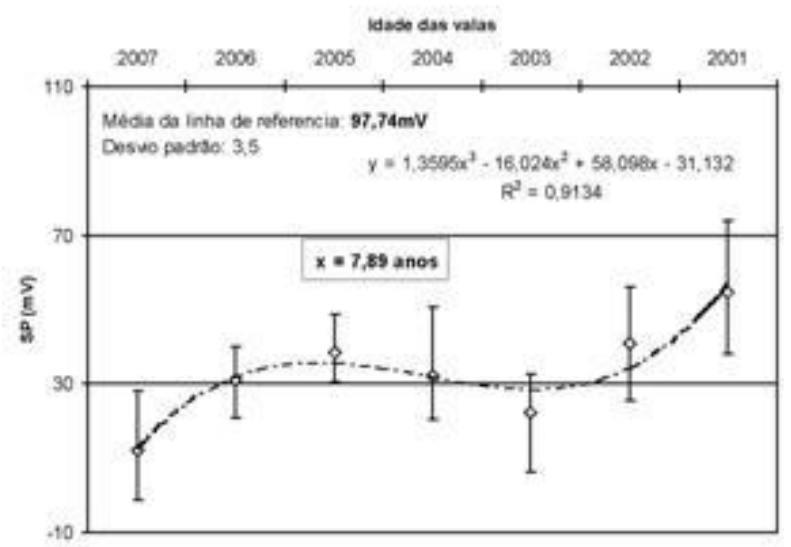

Figura 5 - Analise estatística dos dados da segunda fase. 


\section{Conclusões}

Os resultados revelam a possibilidade de aplicação do método geofísico de Potencial Espontâneo em estudos de áreas de disposição de resíduos sólidos domiciliares, seja em estudos preliminares em áreas sob ausência de dispositivos de monitoramento ambiental, seja como técnica complementar de investigação.

As medidas de potencial elétrico natural indicam uma relação com o tempo de decomposição dos resíduos, ou seja, gradativo aumento do potencial elétrico concomitante ao aumento do período de residência dos resíduos. A ocorrência de valores próximos a zero na vala de 2007 e valores positivos e crescentes no sentido das valas mais antigas indicam que a decomposição de resíduos orgânicos produz potencial elétrico passível de medição pelo instrumental geofísico.

O crescente aumento dos valores de potencial elétrico possibilitam uma análise estatística previsional, que indica a necessidade de aproximadamente 8 anos para que os valores medidos sobre as valas de resíduos sejam semelhantes ao potencial elétrico da área adjacente não contaminada.

Este resultado é um indicativo do tempo médio de consumo de grande parte da matéria orgânica contida em resíduos gerados em domicílios. Contudo, a decomposição de resíduos como papéis, plásticos e metais demandam dezenas a centenas de anos, sendo que a possibilidade de geração de potencial elétrico neste caso é algo incerto a aberto a estudos futuros.

O contraste de potencial elétrico natural detectado entre a área de disposição de resíduos sólidos e o terreno em redor, sensibilidade do instrumental geofísico e relação direta entre potencial elétrico e tempo de residência de resíduos orgânicos, constituem fatores que justificam a aplicação deste método de forma sistemática em estudos de monitoramento de áreas de disposição de resíduos sólidos que contenham matéria orgânica.

\section{Referências}

BAGCHI, A. Natural attenuation mechanisms of landfill leachate and effects of various factors on the mechanism. Waste Management Research, no 5, p. 453-464, 1987.

CORWIN, R. F. The self-potential for environmental and engineering applications. In: Geotechnical and Environmental Geophysics, Society of Exploration Geophysics, Tulsa, USA, Investigations in Geophysics, no 5,1990 , p.127-145.

LANDIM, P. M. B. Análise estatística de dados geológicos. São Paulo: Edunesp. $2^{\circ}$ ed., 2004. 253p.

MOREIRA, C. A.; BRAGA, A. C. O. \& FRIES, M. Degradação de resíduos e alterações na resistividade elétrica, pH e Eh. Revista Brasileira de Geofísica, vol. 27, no 2, p.283 - 293, 2009.

NAUDET, V.; REVIL, A.; BOTTERO, J. Y.; BÉGASSAT, $P$. Relationship between self-potential (SP) signals and redox conditions in contaminated groundwater.
Geophysical Research Letters, vol. 30, no 21, p. 1-4, 2003.

NAUDET, V.; REVIL, A.; RIZZO, E.; BOTTERO, J. Y.; BÉGASSAT, P. Groundwater redox conditions in a contaminant plume from geoelectrical investigations. Hydrogeology and Earth System Sciences, vol. 8, no 1, p.8-22, 2004.

ORELLANA, E. Prospeccion Geoelectrica en Corriente Continua, Biblioteca Técnica Philips, Madrid: Paraninfo, 523 p, 1972.

PETIAU, G. Second generation of Lead-Lead Chloride Electrodes for Geophysical applications. Pure and Applied Geophysics, № 157, p. 357-382, 2000. 